\title{
Mortality trends for ischemic heart disease in China: an analysis of 102 continuous disease surveillance points from 1991 to 2009
}

Xia Wan ${ }^{1+}$, Hongyan Ren ${ }^{2 \dagger}$, Enbo $\mathrm{Ma}^{3}$ and Gonghuan Yang ${ }^{1 *}$

\begin{abstract}
Background: In the past 20 years, the trends of ischemic heart disease (IHD) mortality in China have been described in divergent claims. This research analyzes mortality trends for IHD by using the data from 102 continuous Disease Surveillance Points (DSP) from 1991 to 2009.

Method: The 102 continuous DSP covered 7.3 million people during the period 1991-2000, and then were expanded to a population of 52 million in the same areas for 2004-2009. The data were adjusted by using garbage code redistribution and underreporting rate, mapped from international classification of diseases ICD-9 to ICD-10. The mortality rates for IHD were further adjusted by the crude death proportion multiplied by the total number of deaths in the mortality envelope, which was calculated by using $\log _{\mathrm{t}}=\mathrm{a}+\mathrm{bt}$. Age-standard death rates (ASDRs) were computed using China's 2010 census population structure. Trend in IHD was calculated from ASDRs by using a joinpoint regression model.
\end{abstract}

Results: The IHD ASDRs increased in total in regions with an average annual percentage change (AAPC) 4.96\%, especially for the Southwest (AAPC $=7.97 \%$ ) and Northeast areas (AAPC $=7.10 \%$ ), and for male and female subjects (with 5\% AAPC) as well. In rural areas, the year 2000 was a cut-off point for mortality rate with annual percentage change increasing from 3.52\% in 1991-2000 to 9.02\% in 2000-2009, which was much higher than in urban areas (AAPC $=1.05 \%)$. And the proportion of deaths increased in older adults, and more male deaths occurred before age 60 compared to female deaths.

Conclusion: By observing a wide range of areas across China from 1991 to 2009, this paper concludes that the ASDR trend for IHD increased. These trends reflect changes in the Chinese standard of living and lifestyle with diets higher in fat, higher blood lipids and increased body weight.

Keywords: Ischemic heart disease, Mortality rate, Disease surveillance points

\section{Background}

Ischemic heart disease (IHD) was the leading cause of death globally in the past two decades. In 2013, it caused as many deaths as chronic obstructive pulmonary disease (COPD), diabetes, cirrhosis, lung cancer, and liver cancer combined [1]. The mortality trend of IHD is one of the

\footnotetext{
* Correspondence: yangghuan@vip.sina.com

${ }^{\dagger}$ Equal contributors

'Institute of Basic Medical Sciences, Chinese Academy of Medical Sciences and School of Basic Medicine, Peking Union Medical College, No. 5 Dong Dan San Tiao, Dongcheng District, Beijing 100005, China

Full list of author information is available at the end of the article
}

key indicators closely related to the global target of prevention and control of non-communicable diseases [2].

China, with its rapid economic development over the past 20 years, is experiencing a huge disease burden caused by cardiovascular diseases [3]. In 2012, cardiovascular disease (CVD) claimed 3.5 million lives in China, $40 \%$ of total deaths [4]. In China, there are two national surveillance systems, one is Disease Surveillance Points (DSP) system administered by Chinese Center for Disease Control \& Prevention (CCDC) and the other is the sample vital registration (VR) system administered 
by National Health and Family Planning Commission (NHFPC) of the People's Republic of China. The Report on Cardiovascular Diseases in China (2016, [5] showed that the IHD mortality rate increased from 2002 to 2015 by using data from NHFPC. Most studies from DSP also showed that the trend of age-standardized death rate (ASDR) for IHD in China has been increasing [6, 7]. Even in some cities of China, such as Beijing [8] and Tianjin [9], where the prevention strategies for CVD were implemented relatively well, the mortality rates of IHD still increased. But two newly published Global Burden of Disease (GBD) studies showed diverse results. GBD 2010 results showed that in China the ASDR for IHD increased from 55.7 per 100,000 in 1990 to 70.1 per 100,000 in 2010 [3]. While GBD 2013, using the same data sources, showed a different mortality trend for IHD in China, which remained stable over the past two decades, with 115.40 per 100,000 in 1990 and 115.89 per 100,000 in 2013 [10]. So, over the past 20 years in China, has the mortality rate for IHD increased or has it leveled off? Someone argued that the different mortality trends in IHD in the two studies is owing to covering different populations with adjustment to the disease surveillance system since 2000 [11].

In order to provide further evidence and determine the IHD mortality trends for policy-makers to create timely strategies for CVD prevention and control, this study examined the distribution and trends of IHD mortality in modern China by sex, age, area of residence (urban versus rural), and seven regions (Northeast, North, East, South, Central, Northwest and Southwest) of China, based on a relatively fixed population group for 102 continuous DSP during the past 20 years.

\section{Methods}

\section{Data sources}

The DSP system for recording causes of death was established in China in 1990, covering a population of 10 million people in 145 locations in all provinces by utilizing multiple-stratified random sampling [12]. The system was expanded in 2001 to cover 71.4 million people. The expanded DSP system was adjusted to cover 161 locations which included 103 of the original locations and 58 new locations [11]. The adjusted DSP system expanded to cover the whole district of a city or county, instead of one or two residential district(s) or town(s) at each location.

The system came almost to a standstill from 2001 to 2003 because of DSP adjustments. So our research analyzed 102 continuous DSP from 1991 to 2000 provided by Institute of Basic Medical Sciences at Chinese Academy of Medical Sciences and from 2004 to 2009 (one point was deleted because of missing data for 5 continuous years) provided by CCDC, covering 7.3 million people in 1991-2000 and expanded to a population of 52 million in the same areas for 2004-2009. Before 2000, 1.3 million subjects were from urban areas and 6 million lived in rural areas. After 2004, due to the strong trend in urbanization, the sample population in the 102 continuous DSP points included 17 million people in urban areas and 35 million in rural areas. When considering the data according to the seven regions, which were created based on the population and area; East areas included the most points, with 26 points that expanded from covering a surveillance population of 2.2 million in the original set to a 13.4 million surveillance population. The Northeast has the fewest points (11) with the lowest population (growing from 0.6 million to 6 million). More details on the characteristics of the 102 DSP points can be found in Additional file 1 and the map of DSP in Fig. 1.

\section{Data collection and data quality}

At each DSP site, there was at least one township hospital, and the 'Disease Prevention Unit' in these hospitals was responsible for vital registration. In urban areas, almost half of all deaths occurred in health facilities, and there were standard protocols for death registration that were closely adhered to. The DSP staff collected the death certificate from the hospital, completed by the physician who attended the death. For deaths occurring at home, a staff member from the Unit visited the household, and completed a death certificate based on a description of symptoms from family members (which followed the standardized verbal autopsy procedure), and on available documents from recent contact with health services. The proportion of causes of death by the verbal autopsy (VA) approach was about 7\% [13]. Validity of the VA procedure for adult deaths in China has been assessed in another paper [14]. The detailed working procedure for mortality registration is also described in other works [12, 15, 16]. During 1991-1995, about $42 \%$ of CVD death cases were diagnosed at hospitals classified as county-level or above [17], and the proportion kept increasing, reaching $56 \%$ in 2000 [18]. After 2000 , more than $75 \%$ of deaths were diagnosed from county-level hospitals or above [19]. The proportion of deaths from cardiovascular diseases diagnosed by clinical and physicochemical means (including blood biochemical examination, electrocardiogram, chest X-ray, even coronary artery Computed Tomography and brain angiography, etc.) was about $60 \%$ [19]. In general, the quality of data collection in DSP is high, and this has been validated in other previous studies [20-22]. 


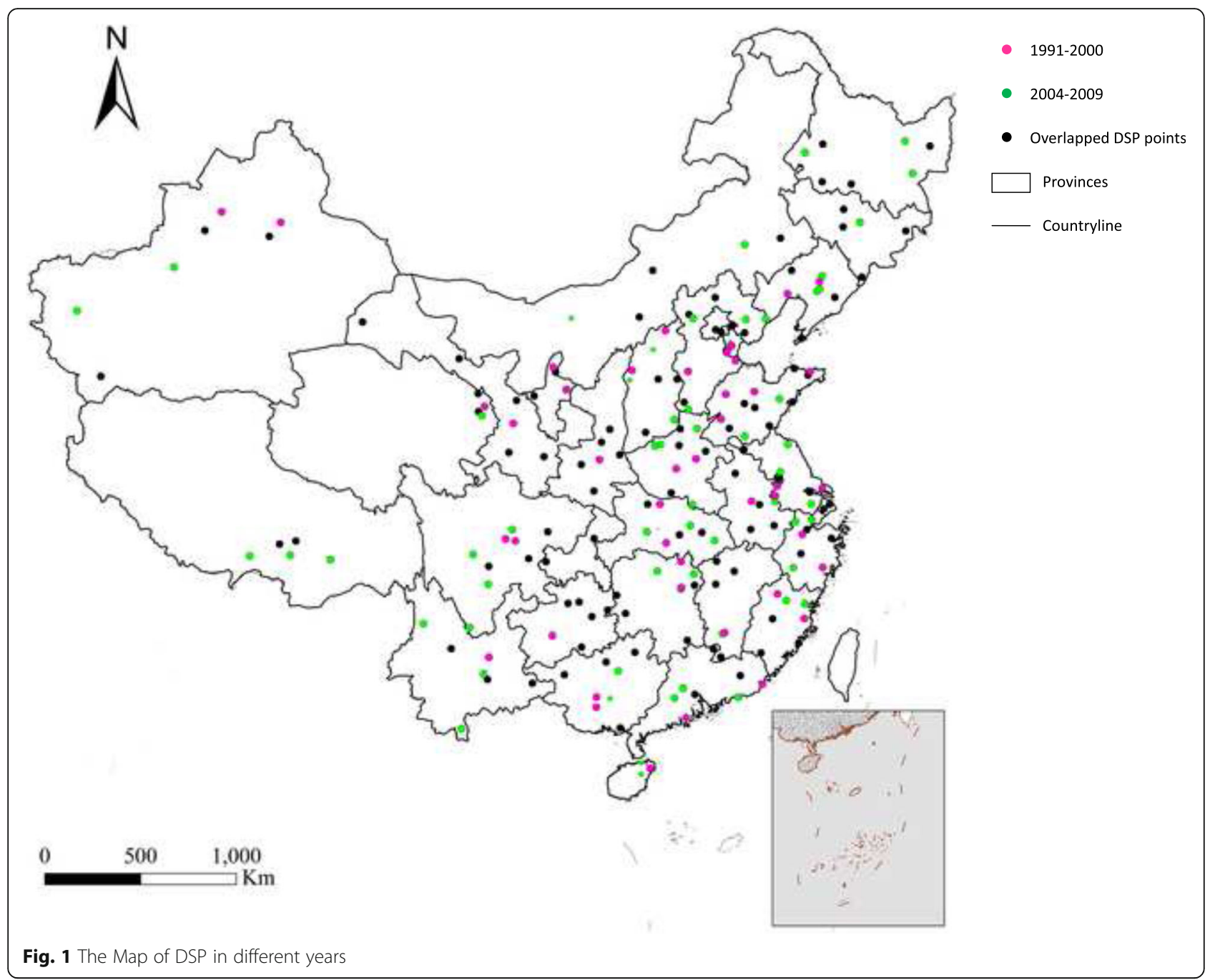

\section{Data analysis}

\section{Redistributing garbage codes}

In 1996, the GBD study introduced the term "garbage coding" for the practice of assigning deaths to causes that are not useful for public health analysis of cause-ofdeath data as part of the assessment of the GBD [23]. This study has used the term garbage code (GC) to refer to all deaths assigned to codes that should be redistributed to enhance the validity of public health analysis. Therefore, certain codes were not regarded as the underlying cause of death, including senility (ICD9-797, ICD10-R54, 1.35\%); atherosclerosis (ICD9-440, ICD10I70, 0.50\%); cerebral atherosclerosis (ICD9-437.0, ICD10-I67.2, 0.57\%); and chronic pulmonary heart disease (CPHD, ICD9-416, ICD10-127.9, 6.43\%). These were labeled garbage. Since the proportion of most garbage codes was small and stable, we did not redistribute them into other categories. The only exception was deaths caused by CPHD, which were classified as having an underlying cause of COPD, since in China $96 \%$ of death cases of CPHD developed from COPD [24]. We did not redistribute any other diseases to IHD.

\section{Code mapping}

The codes for cause of death reported from 1991 to 2000 for DSP data with ICD-9 [25] were shifted to ICD10 [26] in order to keep them identical and comparable with the causes as classified after 2004. The ICD codes for IHD are 410-414 in ICD-9 and I20-I25 in ICD-10.

\section{Mortality rate adjustment and analysis}

Every three years from 1991 to 2000 and 2006 to 2008, an independent survey based on "capture-mark-recapture" methods was conducted to estimate under-reporting, and mortality estimates were adjusted accordingly [12, 17, 27]. The average under-reporting rates for 1991 to 2000 and 2006 to 2008 were $13 \%$ [28] and 17\% [27], respectively. Data from DSP in 2004-05 were verified by the national death survey in 2006 [6]. So we used the average rate from the 2006 survey as the rate for 2005 . In addition, based on 
the underreported adjusted rates from 1991 to 2000 and 2006-2009, the envelope of mortality rates for each year were estimated by using the regression model $\log r_{\mathrm{t}}=\mathrm{a}+\mathrm{bt}$, where $r_{t}$. denotes the mortality rate at time point $t$. Trend b was estimated from the logarithm $\left(\log r_{t}\right)$ of the annual event rates using Poisson Regression [29, 30] In order to verify the increasing and decreasing trends of these diseases, ASDRs for each year were computed based on China's 2010 census population structure [31].

Trend in IHD was calculated from ASDRs by the joinpoint regression model (desktop version: Version 4.3.1.0 [32]) for the total surveillance population, the male/female categories, the urban/rural categories, and the seven regions. Default maximum number of joinpoints was 2 . Monte Carlo simulation, with the number of permutations set to 4499 , is used to calculate the permutation $p$ value for each hypothesis set. This analysis compared models by starting with no joinpoints and subsequently testing whether 1 or more joinpoints needed to be entered into the model to best fit the data. The most parsimonious models were selected to report the estimated annual percent change (APC) for each time segment detected and the average annual percent change (AAPC) for only 0 joinpoint or for the full study period, along with the estimated models. The terms increasing or decreasing were used to describe the trend when the APC or AAPC was statistically significantly different from 0; otherwise, the term stable was used. All significance tests were 2-sided. Statistical significance was defined as $P<0.05$.

Most IHD deaths took place after age 40, therefore we restricted the death proportion to ages 40-49, 50-59, $60-69,70-79$ and 80 and over, separately, by sex. Since the surveillance population in each point was not large, the average 5-year (for 1991-1995, 1996-2000), 2-year (for the 2004-2005 survey) or 4-year (for 2006-2009) deaths were used for observation of each DSP point, which helped make numbers more stable.

\section{Results}

The estimation equation on ASDR for IHD by year was $\log r_{\text {t. }}=-95.189922+0.049597 \times t$, and AAPC was $5.08 \%$ with statistical significance. Both male and female mortality rates increased by a similar speed (AAPC $=5 \%$ ) (Fig. 2). The number of men dying from IHD was consistently higher than the number of women during this time period, which was about $33.3 \%$ more male than female deaths annually.

The increasing trends in urban and rural areas were also statistically significant. However, the increasing trend in urban areas (Urban: $\log r_{\mathrm{t} .}=-16.531373+0.010404 \times \mathrm{t}$ ) was not faster than rural areas, with AAPC $1.05 \%$ and $6.2 \%$, respectively. In urban areas, the rate for males fluctuated a lot (the estimated regression model had no statistical significance), so the rate for females was responsible for the increase. While in rural areas, the year 2000 was a cut-off point for mortality rate with APC increasing from $3.52 \%\left(\log r_{\mathrm{t} .}=-65.372493+0.034575 \times \mathrm{t}\right)$ in $1991-$ 2000 to $9.02 \%\left(\log r_{t .}=-168.972238+0.086375 \times \mathrm{t}\right)$ in 2000-2009, as well as for male mortality rate (Fig. 3).

In terms of the seven regions, the mortality rates were low in the south areas and high in the north areas. From 1991 to 2009, the mortality rates increased in the Northeast, North, Northwest and Southwest for the whole period, especially in the Southwest (AAPC $=7.97 \%)$ and Northeast (AAPC $=7.10 \%$ ). For the rest of the areas, the mortality rates increased in the East during 1991-1996, in the South during 1991-2000 and in Central areas during 1991-2005 with statistical significance, and showed a stable or decreasing trend for other time periods without statistical significance (Fig. 4).

For the proportion of deaths after age 40, at ages 40-59 and $70-79$, the death proportion remained fairly stable, but the proportion for ages 60-69 reduced with that of ages above 80 years old increasing. Compared to female deaths, more male deaths took place before age 60 (male: about 20\%, female: about 10\%). For ages 60-69, the male proportion reduced from $25.9 \%$ to $17.8 \%$ and female reduced from $20.2 \%$ to $12.8 \%$ from 1991 to 2009 (Fig. 5).

\section{Discussion}

In analyzing areas in China from 1991 to 2009, the ASDR trend for IHD increased substantially for men and women, urban and rural, and in most regions. The speed of increase of ASDR varied greatly between regions of the country and between urban and rural areas. The greater increase in the Northeast and Southwest widened the gap between different regions. Of course, we couldn't know the situation in other areas in China, if there were an extreme scenario where IHD mortality rate decreased in all other areas in the past 20 years, the trend of IHD mortality rate across the whole country could decrease. But from these 102 continuous DSP, the ASDR trend for IHD should increase.

Actually, the increasing mortality trend for IHD in China matched the related changing levels of prevalence of the various risk factors. In Western countries, it has been shown that IHD is related to high serum total cholesterol (TC) levels [33-35]. With growing urbanization and industrialization in Asia, the same relationship has been shown between $\mathrm{TC}$ and IHD [36]. An earlier study focused on Shanghai found a strongly positive, and apparently independent, relation between serum cholesterol concentration and death from IHD [37]. Also a study in Beijing found agestandard IHD mortality increased from 1984 to 1999, which can be explained by rises in TC, reflecting an increasingly "Western" diet [38]. 


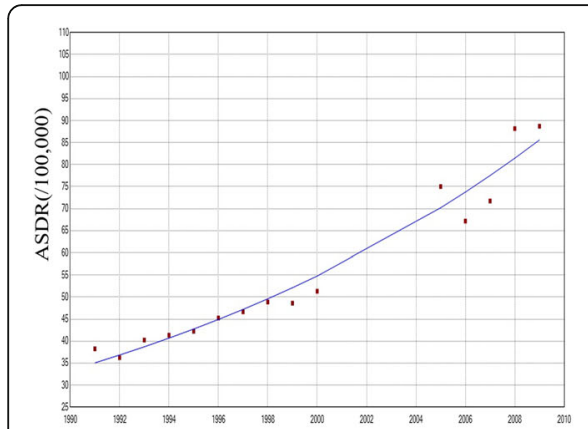

a Tota1

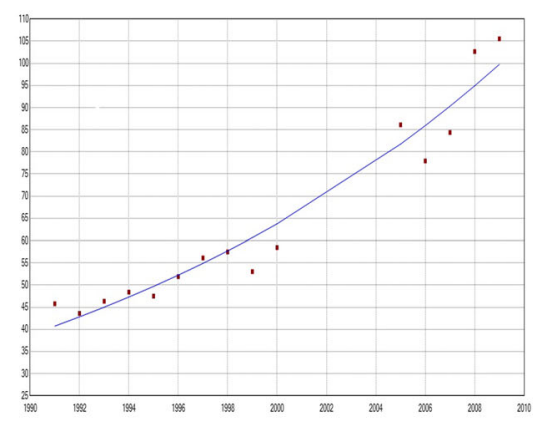

b Male

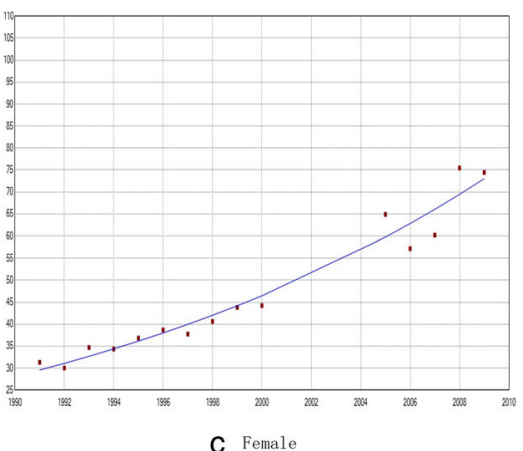

C Female

a) Total: $\log r_{t}=-95.189922+0.049597 \times t \quad$ APC $=5.08$

b) Male: $\log r_{t}=-95.355083+0.049755 \times t \quad$ APC $=5.10$

$\begin{array}{ll}\text { c) Female: } \log \mathrm{r}_{\mathrm{t}}=-96.634577+0.050237 \times \mathrm{t} & \text { APC }=5.15\end{array}$

Fig. 2 Trend of ASDR of IHD from 1991 to 2009 by sex $(/ 100,000)$

In China, over the past 20 years, cereals decreased as a proportion of the diet, whereas fats increased fast. From 1982 to 2002, fat intake increased from $68.3 \mathrm{~g}$ to $85.5 \mathrm{~g}$ per day per person in urban areas and increased from $39.6 \mathrm{~g}$ to $72.7 \mathrm{~g}$ per day per person in rural areas. Although there was an even higher fat intake in urban areas, it increased faster in rural areas [39]. Other studies in Beijing confirmed the trend that the total fat intake increased from $334.5 \mathrm{~g} / \mathrm{d}$ to $488.4 \mathrm{~g} / \mathrm{d}$ in urban areas and from $124.8 \mathrm{~g} / \mathrm{d}$ to $350.7 \mathrm{~g} / \mathrm{d}$ in rural areas from 1983 to 2002, where it increased faster in rural areas $[40,41]$. These diet risk factors give rise to an increased proportion of the population that is overweight and obese. In 2010, the national overweight and obese prevalence for adults was $30.6 \%$ and $12.0 \%$, respectively [42], which also caused increasing levels of TC [43].

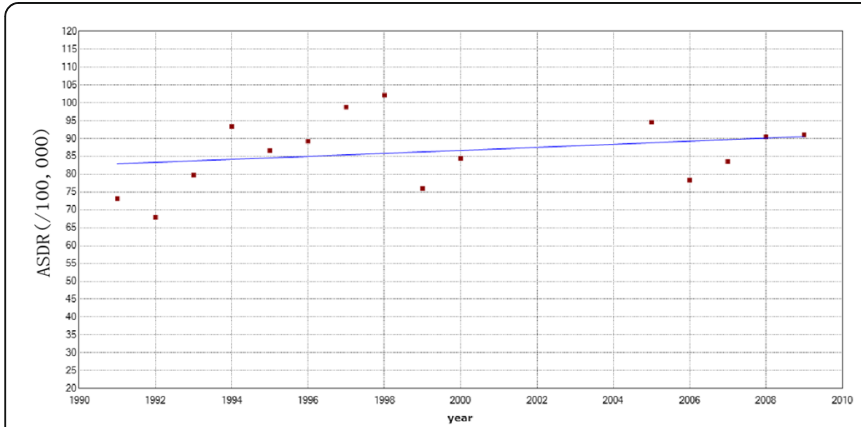

a Urban male

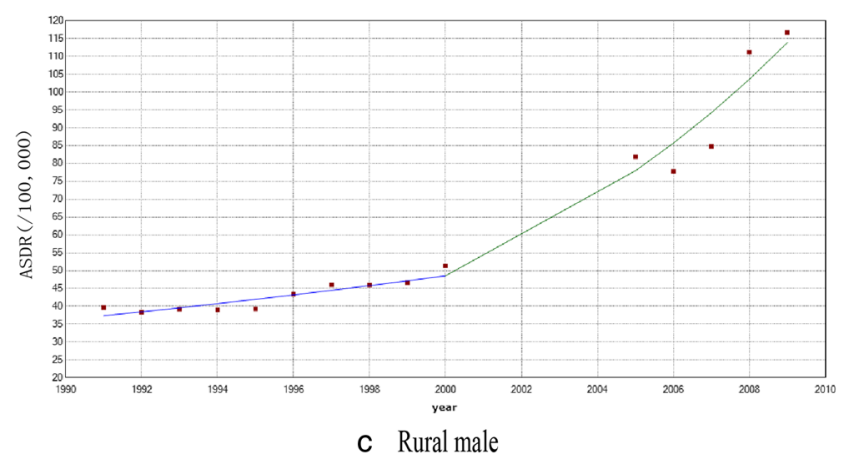

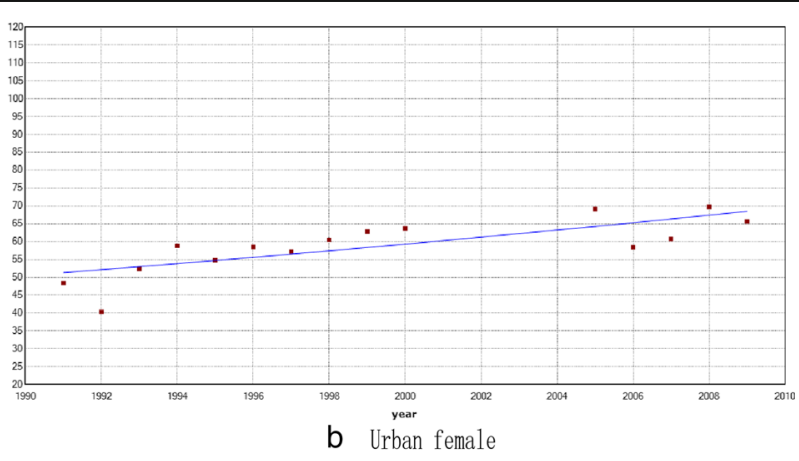

b Urban female

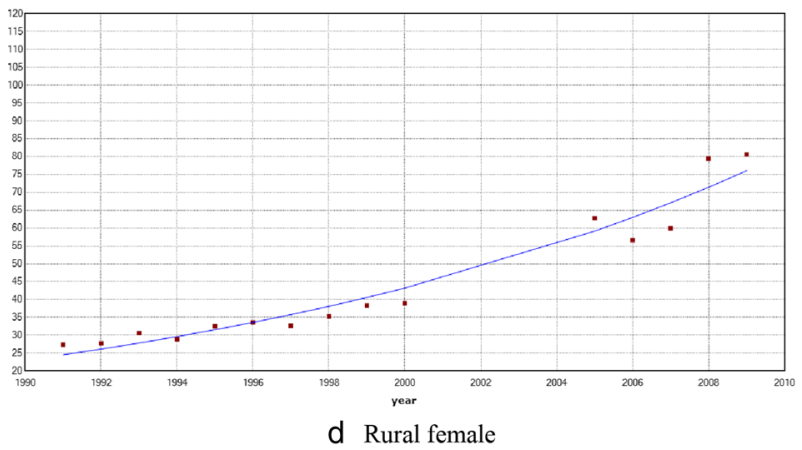

a) Urban male: $\log r_{t}=-5.317184+0.004890 \times t^{*} \quad$ AAPC $=0.49$

b) Urban female: $\log r_{t}=-27.994704+0.016038 \times t \quad$ AAPC $=1.62$

c) Rural male (1991-2000): $\log r_{r}=-54.400518+0.029142 \times t \quad$ APC $=2.96$

Rural male (2000-2009): $\log r_{t}=-185.522086+0.094702 \times t \quad A P C=9.93$

Rural male: AAPC $=6.4$

d) Rural female: $\log r_{t}=-121.870527+0.062818 \times t \quad$ AAPC $=6.48$

Note: * the estimated regression model had no statistical significance

Fig. 3 Trend of ASDR for IHD from 1991 to 2009 by sex and urban/rural areas $(/ 100,000)$ 


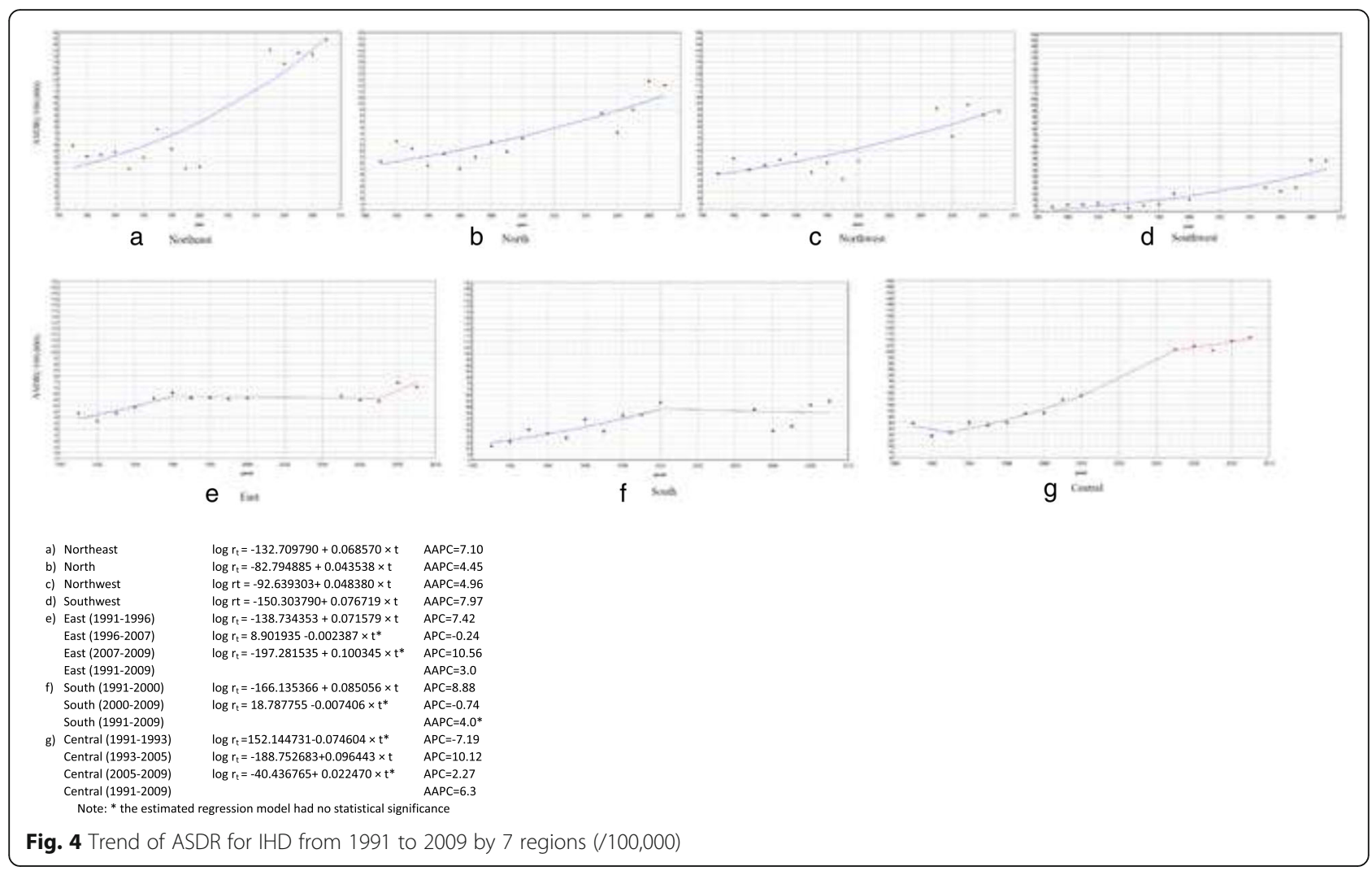

Thus the TC increase changes the pattern of IHD mortality and morbidity. That's why the mortality rate in rural areas increased faster.

In addition, in our study, the number of men dying from IHD was higher than the number of women, and also the mortality rate increased faster in Northeast China. This pattern could also be seen in the MONICA study [44] and GBD 2013 [10], which was also associated with risk factors. Hypertension, diabetes, hypercholesterolemia and smoking are major risk factors for IHD [45]. From the 1980s to mid 1990s, blood pressure and overweight values increased significantly, in the north higher than in the south, in males higher than females [46]. And the explanation for more male deaths than female ones could be due to the high levels of tobacco consumption in men (52.1\%) compared with women (2.7\%)

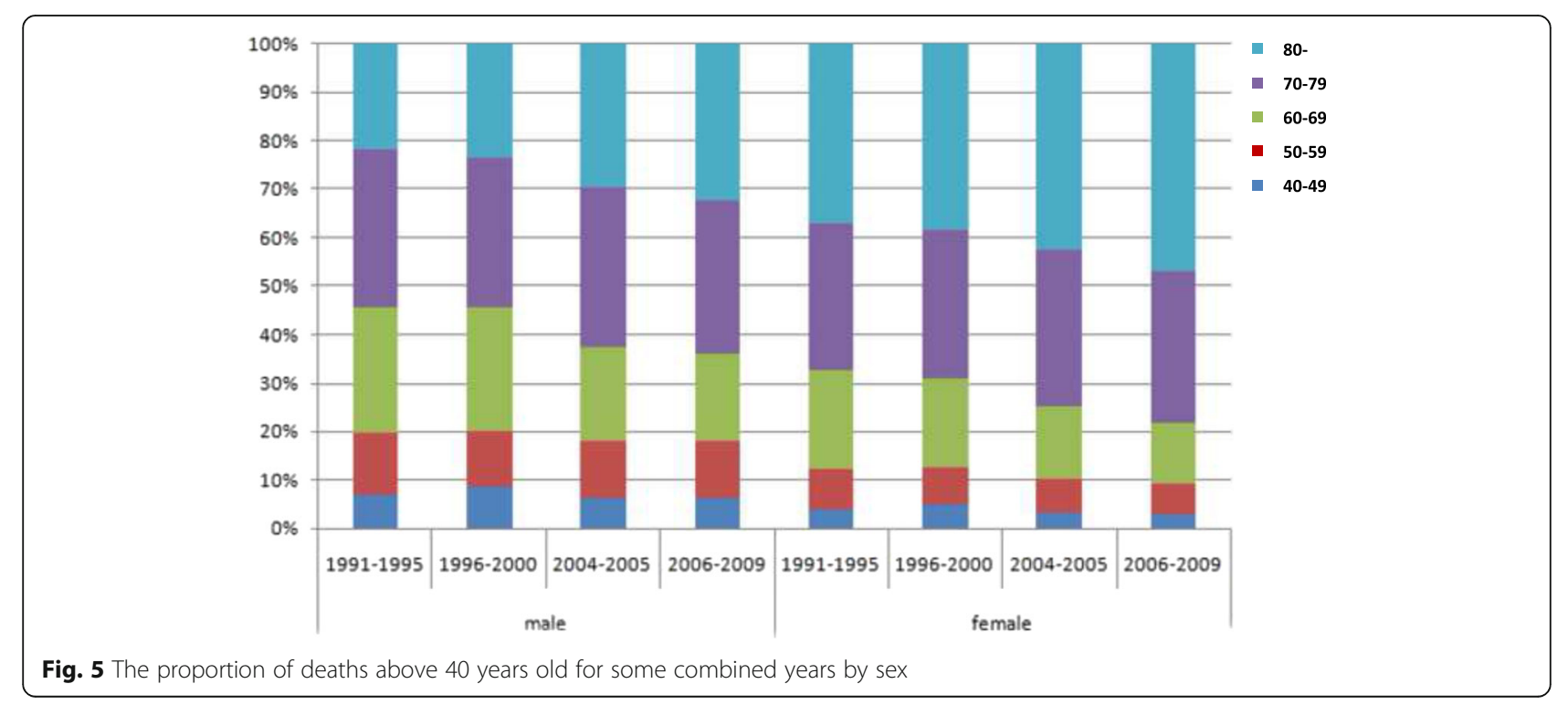


in China [47]. Higher smoking rates were also seen in the northeast and northern parts of China [48].

Jiang, et al. (2011) showed that a large number of IHD patients took place at ages above 60 , but the incidence of young people increased [49]. In addition, male IHD incidence rate increased $30.3 \%$ among the 35-44 age group and increased $21 \%$ among the $45-55$ age group within 3 years [50]. From our study, the proportion of deaths at ages 40-59 remained stable with that of ages above 80 years old increasing, which has not shown more and more IHD deaths in young adults as yet, maybe because of medical treatment improvement and life of expectancy of Chinese people increasing. Therefore, not that many young adults died, but the deaths after age 80 could increase.

In this study, since the actual rates fluctuated in some years, the predicted mortality rate for each year calculated by the Poisson regression models was used, instead of the actual rate. This idea comes from GBD research. Before analysis, GBD research usually used models to estimate the envelope to further adjust the mortality rates [51]. Although the predicted mortality for each year would be affected by the mortality or deaths in other years, we think the predicted values are good for trend testing. The data from 102 continuous DSP covered only one street or one block before 2000, and then were expanded to cover a whole district or county, which may have caused some bias. Fortunately, the data collected included a large number of people (7.3 million before the year 2000 and 52 million for 2004-2009) and almost covered 31 out of 33 provinces/municipalities. Furthermore, before year 2000 it was randomly selected from each area and the demographic character (e.g. age structure, lifestyle behavior, etc.) of each point was similar to those after 2000, which means the samples could represent the whole and are relevant for comparison. So we can assume these samples, coming from the relative fixed population or the same primary sampling units (PSUs), should have the lowest variance for estimated difference for a variable of interest from repeated surveys from a statistical point of view.

In another paper we already found the reason that caused the trend decrease in GBD 2013 - chronic pulmonary heart disease as GC redistribution [52]. Before 2000, in the mortality database of DSP, there was a corresponding proportion of chronic PHD. In GBD 2010, the death cases of chronic PHD were totally redistributed into COPD, which was the same as the results from the original methods in the DSP system, as well as this study. But the cases were redistributed into COPD, IHD and hypertension heart disease by different fractions based on the module in GBD 2013. COPD is the major cause of chronic PHD, and probably accounts for $80-90 \%$ of the causes from western literature [53].
Studies in China testified that the proportion was even higher for Chinese. Fu et al. (1988) investigation confirmed that $96.6 \%$ of Chronic PHD was from COPD in Beijing [24]. Owing to changing the redistribution fraction of chronic PHD, more IHD cases were adjusted in the early 1990s. So that's why GBD 2010 and our study's results showed that the mortality trend increased, while GBD 2013 results showed the trend remained stable. Therefore, in GBD 2015, using the same GC redistribution strategy of GBD 2010, the trend increased in China. So in a nutshell, the increased mortality trend for IHD could describe the real world for Chinese people. From this paper, it is indicated that in the past two decades the ASDR trend for IHD has increased by an estimated annual 1.2 million deaths, which has caused a challengeable burden of diseases. Therefore, control of risk factors in China is an urgent task, including controlling tobacco use and promoting healthy diets, which could lower death rates and avoid increased medical expense.

\section{Limitations}

While this paper utilizes extensive and wide-ranging data, this data does have some limitations. The data used in this paper only covers 1991 to 2009 and thus does not reveal more recent trends or patterns. However, the time period studied captures the critical period of transition in Chinese society and the economy. The data from continuous DSPs covered only one street or one block before 2000, and then were expanded to cover a whole district or county; hence a sensitivity analysis should be done. As a result, most of the cases of death's home address was not described in detail, so it is hard for us to identify those cases of deaths whose home address was in the streets or blocks which were both active in both periods. That's one of the limitations in this study. Another limitation in this study is that we did not take the sampling weights and strata into account.

\section{Conclusions}

By observing a wide range of areas across China from 1991 to 2009, this paper concludes that the ASDR trend for IHD has increased. These trends reflect changes in the Chinese standard of living and lifestyle with diets higher in fat, higher blood lipids and increased body weights. Therefore, control of risk factors in China is an urgent task to lower IHD death rates and avoid increased medical expense.

\section{Additional file}

Additional file 1: The Characteristics of 102 DSP Points. There were four variables in this table, including the DSPs name, regions where each DSP belongs to, population for each DSP in 1991 and 2009 respectively. (DOCX $49 \mathrm{~kb}$ ) 


\section{Abbreviations}

AAPC: Average Annual Percentage Change; APC: Annual Percent Change; ASDRs: Age-standard Death Rates; CCDC: Chinese Center for Disease Control \& Prevention; COPD: Chronic Obstructive Pulmonary Diseases; CPHD: Chronic Pulmonary Heart Disease; CVD: Cardiovascular Disease; DSP: Disease Surveillance Points; GBD: Global Burden of Disease; GC: Garbage Code; IHD: Ischemic Heart Disease; NHFPC: National Health and Family Planning Commission; PSUs: Primary Sampling Units; TC: Total Cholesterol; VA: Verbal Autopsy; VR: Vital Registration

\section{Acknowledgments}

The authors thank the Chinese Center for Disease Control and Prevention for providing us with the data for 2004 to 2009.

\section{Funding}

This study was funded by a China Medical Board Grant on CMB-CP in Burden of Diseases in China (12-107, 15-208); China Medical Board Faculty Development Award [grant number G16917561]; and China Scholarship Council [grant number 201408110083]

\section{Availability of data and materials}

The data that support the findings of this study are available from CCDC and CAMS. The dataset from 1991 to 2000 used and analyzed during this study from CAMS are available from the corresponding author on reasonable request. The data after 2000 that support the findings of this study are available from CCDC but restrictions apply to the availability of these data, which were used under license for the current study, and so are not publicly available. Data are however available from the authors upon reasonable request and with permission of CCDC.

\section{Authors' contributions}

$\mathrm{XW}$ interpreted the findings and was a major contributor in writing the manuscript. HYR and EM contributed to the interpretation of the results. GHY was involved in the design and coordination of the study and revision of the manuscript. All authors read and approved the final manuscript.

\section{Ethics approval and consent to participate}

This study didn't conduct data collection, and just analyzed the existed surveillance data provided by CCDC and Institute of Basic Medical Sciences, Chinese Academy of Medical Sciences (CAMS). And the death cases' name and ID couldn't be identified in the dataset. So it is not applicable for ethics approval and consent to participate.

\section{Consent for publication}

Not applicable.

\section{Competing interests}

The authors declare that they have no competing interests.

\section{Publisher's Note}

Springer Nature remains neutral with regard to jurisdictional claims in published maps and institutional affiliations.

\section{Author details}

${ }^{1}$ Institute of Basic Medical Sciences, Chinese Academy of Medical Sciences and School of Basic Medicine, Peking Union Medical College, No. 5 Dong Dan San Tiao, Dongcheng District, Beijing 100005, China. ${ }^{2}$ State Key Laboratory of Resources and Environmental Information System, Institute of Geographic Sciences and Natural Resources Research, Chinese Academy of Sciences, No.11 Datuan Load A, Chaoyang District, Beijing 100101, China. ${ }^{3}$ Faculty of Medicine, University of Tsukuba, 1-1-1 Tennodai, Tsukuba 305-8575, Japan.
Received: 4 February 2017 Accepted: 30 June 2017

Published online: 25 July 2017

\section{References}

1. Shepard DVA, Moran A, et al. Ischemic heart disease worldwide, 1990-2013: estimates from the global burden of disease study 2013. Circ Cardovasc Qual Outcomes. 2015 July:8(4):455-6.

2. WHO. GLOBAL STATUS REPORT on non-communicable diseases 2014 [http://apps.who.int/iris/bitstream/10665/148114/1/9789241564854_eng. pdf?ua=1. (Accessed at Dec. 30, 2015)].

3. Yang G, Wang Y, Zeng Y, Gao GF, Liang X, Zhou M, Wan X, Yu S, Jiang Y, Naghavi M, et al. Rapid health transition in China, 1990-2010: findings from the global burden of disease study 2010. Lancet. 2013;381(9882):1987-2015.

4. Wang Y. Database for cause of death from diseases surveillance system, 2012. Beijing: People's Medical Publishing House; 2013. [in Chinese]

5. National Center for Cardiovascular Disease, China. Report on Cardiovascular Diseases in China (2016). Beijing: Encyclopedia of China Publishing House; 2017. [in Chinese]

6. Yang G, Kong L, Zhao W, Wan X, Zhai Y, Chen LC, Koplan JP. Emergence of chronic non-communicable diseases in China. Lancet. 2008;372(9650):1697-705.

7. Liu MB, Wang W, Zhou MG. Trend analysis on the mortality of cardiovascular diseases from 2004 to 2010 in China. Zhonghua Liu Xing Bing Xue Za Zhi. 2013;34(10):985-8. [in Chinese]

8. Sun JY, Liu J, Xie XQ, Wei ZH, Wang W, Wang M, Qi Y, Liu J, Guo MN, Zhang $X Y$, et al. Surveillance on the incidence of acute coronary events in the permanent residents of Beijing aged 25 years and more from 2007 to 2009. Zhonghua Xin Xue Guan Bing Za Zhi. 2012;40(3):194-8. [in Chinese]

9. Jiang G, Wang D, Li W, Pan Y, Zheng W, Zhang H, Sun YV. Coronary heart disease mortality in China: age, gender, and urban-rural gaps during epidemiological transition. Rev Panam Salud Publica. 2012;31(4):317-24.

10. Zhou M, Wang H, Zhu J, Chen W, Wang L, Liu S, Li Y, Wang L, Liu Y, Yin P, et al. Cause-specific mortality for 240 causes in China during 1990-2013: a systematic subnational analysis for the global burden of disease study 2013. Lancet. 2016:387(10015):251-72.

11. Zhou M, Jiang Y, Huang Z, Wu F. Adjustment and representativeness evaluation of national disease surveillance points system. Disease Surveillance. 2010;25(3):239-44. [in Chinese]

12. Yang G, Hu J, Rao KQ, Ma J, Rao C, Lopez AD. Mortality registration and surveillance in China: history, current situation and challenges. Popul Health Metr. 2005;3(1):3.

13. Wang Y. Database for cause of death from diseases surveillance system, 2009. Beijing: Military Medical Science Press; 2012. [in Chinese]

14. Yang G, Rao C, Ma J, Wang L, Wan X, Dubrovsky G, Lopez AD. Validation of verbal autopsy procedures for adult deaths in China. Int J Epidemiol. 2006;35(3):741-8.

15. Dai Z, Zheng X, Qi X, Yang G, Liu P. Disease surveillance - methodology and application. Beijing: Hua Xia Press; 1993. [in Chinese]

16. Chinese Center for Disease Prevention and Control. Guideline of National Disease Surveillance Points System Working Procedure. [https://wenku.baidu.com/view/825f27b619e8b8f67d1cb932.html. (Accessed at April 7th, 2017)]

17. Dept. of Disease Control of the Ministry of Health, Chinese Academy of Prevention Medicine. A Series of Reports on Chinese Disease Surveillance (6): 1995 Annual report on Chinese disease surveillance. In: . Beijing: People's Medical Publishing House. p. 1997. [in Chinese].

18. Dept. of Disease Control of the Ministry of Health, Chinese Academy of Prevention Medicine. A Series of Reports on Chinese Disease Surveillance (10): 1999 Annual Report on Chinese Disease Surveillance; 2000. [in Chinese]

19. Chinese Center for Disease Control and Prevention. Database for cause of death from diseases surveillance system, 2008. Beijing (China: People's Medical Publishing House; 2009. [in Chinese]

20. Rao C, Lopez AD, Yang G, Begg S, Ma J. Evaluating national cause-of-death statistics: principles and application to the case of China. Bull World Health Organ. 2005;83(8):618-25.

21. Rao C, Yang G, Hu J, Ma J, Xia W, Lopez AD. Validation of cause-of-death statistics in urban China. Int J Epidemiol. 2007;36(3):642-51.

22. Wang $L$, Yang GH, Ma JM, Rao C, Wan X, Dubrovsky G, Lopez AD. Evaluation of the quality of cause of death statistics in rural China using verbal autopsies. J Epidemiol Commun H. 2007;61(6):519-26. 
23. Naghavi M, Makela S, Foreman K, O'Brien J, Pourmalek F, Lozano R. Algorithms for enhancing public health utility of national causes-of-death data. Popul Health Metrics. 2010;8(1):1-9.

24. Fu Z, Dong J, Guo B, Liu S, Li J. Study on adjustment of pulmonary heart disease. J Hyg Res (Wei Sheng Yan Jiu). 1988;2:50-1. [in Chinese]

25. World Health Organization: Manual of the International Statistical Classification of Diseases, Injuries and Causes of Death, Ninth Revision: Geneva: WHO; 1977.

26. World Health Organization: International Classification of Diseases and Related Health Problems - Tenth Revision (ICD 10): Geneva: WHO; 1992

27. Wang $L$, Wang $L$, Cai $Y, M a L M$, Zhou MG. Analysis of under-reporting of mortality surveillance from 2006 to 2008 in China. Zhonghua Yu Fang Yi Xue Za Zhi. 2011;45(12):1061-4. [in Chinese]

28. Dept. of Disease Control of the Ministry of Health, Chinese Academy of Prevention Medicine. A Series of Reports on Chinese Disease Surveillance (9): 1998 Annual report on Chinese disease surveillance. Beijing: People's Medical Publishing House. 1999. [in Chinese]

29. Salomaa V, Arstila M, Kaarsalo E, Ketonen M, Kuulasmaa K, Lehto S, Miettinen $\mathrm{H}$, Mustaniemi $\mathrm{H}$, Niemela M, Palomaki $\mathrm{P}$, et al. Trends in the incidence of and mortality from coronary heart disease in Finland, 1983-1988. Am J Epidemiol. 1992;136(11):1303-15.

30. Wu Z, Yao C, Zhao D, Wu G, Wang W, Liu J, Zeng Z, Wu Y. Sino-MONICA project: a collaborative study on trends and determinants in cardiovascular diseases in China, part i: morbidity and mortality monitoring. Circulation. 2001;103(3):462-8.

31. Chinese Statistical Yearbook 2010 [http://www.stats.gov.cn/tjsj/pcsj/rkpc/ 6rp/indexch.htm. (Accessed at Oct. 13, 2014)].

32. National Cancer Institute, Division of Cancer Control \& Population Sciences. [https://surveillance.cancer.gov/joinpoint/index.html. (Accessed Jul. 9, 2016)].

33. Lewis BCA, Sigurdsson G, Mancini M, Farinaro E, Oriente P, Carlson LA Ericsson M, Micheli H, Pometta D. Serum lipoproteins in four European communities in a quantitative comparison. Eur J Clin Investig. 1978:8:165-73.

34. LA S. Inter-relations of lipids and lipoproteins with coronary artery disease mortality in 19 countries. Am J Cardiol. 1986;59:5G-10G.

35. Keys A. Coronary heart disease - the global picture. Atherosclerosis. 1975; 22(2):149-92.

36. Wakugami KIK, Kimura Y, Okumura K, Ikemiya Y, Muratani H, Fukiyama K. Relationship between serum cholesterol and the risk of acute myocardial infarction in a screened cohort in Okinawa, Japan. Jpn Circ J. 1998;62:7-14.

37. Chen Z, Peto R, Collins R, MacMahon S, Lu J, Li W. Serum cholesterol concentration and coronary heart disease in population with low cholesterol concentrations. BMJ. 1991;303(6797):276-82

38. Critchley JLJ, Zhao D, Wei W, Capewell S. Explaining the increase in coronary heart disease mortality in Beijing between 1984 and 1999. Circulation. 2004;110(10):1236-44

39. Wang L. Comprehensive report, Chinese nutrition and health survey in 2002. Beijing: People's Medical Publishing House. 2005. [in Chinese]

40. Pang XH, Jiao SF, Huang L, Duan JL, Ren ZY, Liu ZJ. Nutrition and health status of the Beijing residents. Zhonghua Yu Fang Yi Xue Za Zhi. 2005;39(4):269-72. [in Chinese]

41. Yao CH, Hao EH, Wu YY, Dong L. Dietary survey in Beijing in 1983. Chin Med J. 1985;98(6):439-41. [in Chinese]

42. The results of non-communicable diseases and related risk factors from diseases surveillance system points in 2010. [http://wenku.baidu.com/ link?url=kYidqsX-RSkHUqAXqa4ls5TydACOXLZZESJWoOkolJMZjGL Pafa1WZTZIphV8sTHf92ImyvO27NPIXhHHMVyEIWsQt8DHsIGILCNG9wUyCK. Accessed 11 Nov 2016).

43. Zhao D, Liu J, Wang W, Zeng Z, Cheng J, Liu J, Sun J, Wu Z. Epidemiological transition of stroke in China: twenty-one-year observational study from the Sino-MONICA-Beijing project. Stroke. 2008;39(6):1668-74.

44. Wu X. The current prevalence and trend of coronary heart diseases of Chinese people. Chin J Prev Contr Chron Non-commun Dis. 2003;11(4):1901. [in Chinese]

45. Hata J, Kiyohara Y. Epidemiology of stroke and coronary artery disease in Asia. Circ J. 2013;77(8):1923-32

46. The collaborative study group on trends of cardiovascular diseases in China and preventive strategy: [Current status of major cardiovascular risk factors in Chinese populations and their trends in the past two decades]. Chin J Cardiol 2001, 29(2):74-79.

47. Liang XF, editor. China Adult Tobacco Survey in 2015. Beijing: People's medical publishing house. 2016. [in Chinese]
48. Yang GH, Ma JM, Liu N, Zhou LN. Smoking and passive smoking in Chinese, 2002. Zhonghua Liu Xing Bing Xue Za Zhi. 2005:26(2):77-83. [in Chinese]

49. Jiang D, Zhou J. Analysis of 6295 coronary heart disease patients age pattern. Med Inf. 2011;24(4):1389. in Chinese

50. More and more young IHD patient. http://news.cqnews.net/html/2015-10/ 08/content 35457757.htm. (Access Nov. 7, 2016).

51. Wang H, Dwyer-Lindgren L, Lofgren KT, Rajaratnam JK, Marcus JR, LevinRector A, Levitz CE, Lopez AD, Murray CJ. Age-specific and sex-specific mortality in 187 countries, 1970-2010: a systematic analysis for the global burden of disease study 2010. Lancet. 2012;380(9859):2071-94.

52. Wan $X$, Yang $\mathrm{GH}$. Is the mortality trend of ischemic heart disease by the GBD2013 study in China real? Biomed Environ Sci. 2017;30(3):204-9.

53. Weitzenblum E. Chronic cor pulmonale. Heart. 2003:89(2):225-30.

\section{Submit your next manuscript to BioMed Central and we will help you at every step:}

- We accept pre-submission inquiries

- Our selector tool helps you to find the most relevant journal

- We provide round the clock customer support

- Convenient online submission

- Thorough peer review

- Inclusion in PubMed and all major indexing services

- Maximum visibility for your research

Submit your manuscript at www.biomedcentral.com/submit 